\title{
Antibiotiques et réponse SOS bactérienne
}

> Le rôle des antibiotiques dans l'induction directe de la résistance des bactéries aux antibiotiques est connu pour certaines résistances. De récents travaux indiquent que certains antibiotiques, en ayant un effet sur la réponse SOS bactérienne, réponse globale de réparation des dommages à l'ADN, seraient aussi impliqués dans l'induction, l'acquisition et le transfert de résistances. Nous discutons ici le rôle des antibiotiques dans l'acquisition des résistances via la réponse SOS bactérienne; nous l'illustrons avec divers exemples et montrons que les régulateurs de la réponse SOS pourraient être identifiés comme des cibles possibles de nouveaux antimicrobiens. <

Avec la découverte des antibiotiques, les maladies infectieuses furent rapidement considérées comme définitivement vaincues. C'était sans compter avec la capacité des microorganismes à s'adapter à différentes pressions environnementales [1]. L'utilisation mal adaptée et la surconsommation des antibiotiques qui exercent de fortes pressions de sélection sur les populations microbiennes, que ce soit en santé humaine, animale ou en agriculture, ont ainsi favorisé l'émergence de bactéries qui ont développé des résistances à parfois quasiment tous les antibiotiques ${ }^{1}$. La liste des bactéries multirésistantes (BMR) est en augmentation constante, que ce soit à l'hôpital ou dans un contexte communautaire, ce qui pose un vrai problème de santé publique au niveau mondial. En témoigne l'émergence récente de bactéries productrices de carbapénémases qui résistent à quasiment tous les antibiotiques [2]. De nouvelles classes d'antibiotiques sont nécessaires, mais peu sont actuellement en développement. II devient donc urgent d'identifier de nouvelles approches pour lutter contre la résistance bactérienne aux antibiotiques. Mieux comprendre l'origine, l'évolution et

${ }^{1}$ Voir le numéro thématique de médecine/sciences consacré au résistances des bactéries aux antibiotiques, 2010, vol 26, numéro 11 .

\section{Une voie efficace}

d'acquisition des résistances aux antibiotiques

Sandra Da Re ${ }^{1,2}$, Marie-Cécile Ploy ${ }^{1,2,3}$

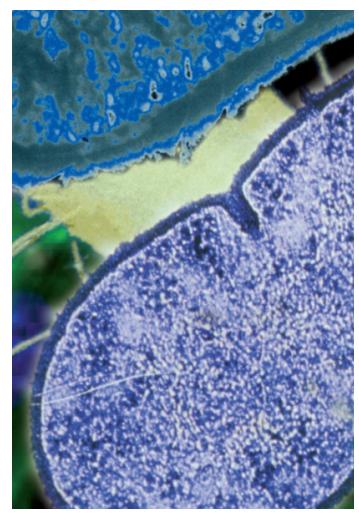

${ }^{1}$ Université de Limoges, UMR-S1092, Limoges Cedex, F-87025, France.

${ }^{2}$ Inserm, U1092,

2 , rue du docteur Marcland, Limoges Cedex, F-87025, France. ${ }^{3} \mathrm{CHU}$ de Limoges, laboratoire de bactériologie-virologie-hygiène, 2, avenue Martin Luther King, Limoges Cedex, F-87042, France. marie-cecile.ploy@unilim.fr

les modes de dissémination des déterminants de cette résistance, mais aussi les réponses physiologiques des bactéries à l'action des antibiotiques, apportera des informations inestimables pour la recherche de nouvelles molécules et/ou de moyens de lutte contre ces résistances.

\section{Acquisition de résistances aux antibiotiques}

La résistance aux antibiotiques peut être naturelle, elle a alors un support chromosomique, ou acquise et dans ce cas, les supports génétiques sont variables. Il peut s'agir : (1) de mutations chromosomiques ponctuelles qui vont par exemple conduire à l'altération de la cible de l'antibiotique (mutations dans les gènes de topo-isomérases pour la résistance aux quinolones, ou dans le gène $r p o B$ pour la rifampicine) ou à des résistances par imperméabilité à l'antibiotique et/ou efflux actif de ce dernier ; (2) d'acquisition de matériel génétique par transfert horizontal de gènes via des éléments génétiques mobiles tels que les plasmides, les éléments intégratifs conjugatifs (ICE) ou les cassettes d'intégrons.

Divers travaux ont montré le lien entre traitement antibiotique (en ville ou à l'hôpital) et acquisition de résistances aux antibiotiques par les populations bactériennes commensales et pathogènes [3], les antibiotiques entraînant la sélection des bactéries ayant acquis un mécanisme de résistance. Cependant, il a été montré que les antibiotiques pouvaient aussi directement susciter les résistances, en induisant leur expression ou leur acquisition. Ainsi, le système à 


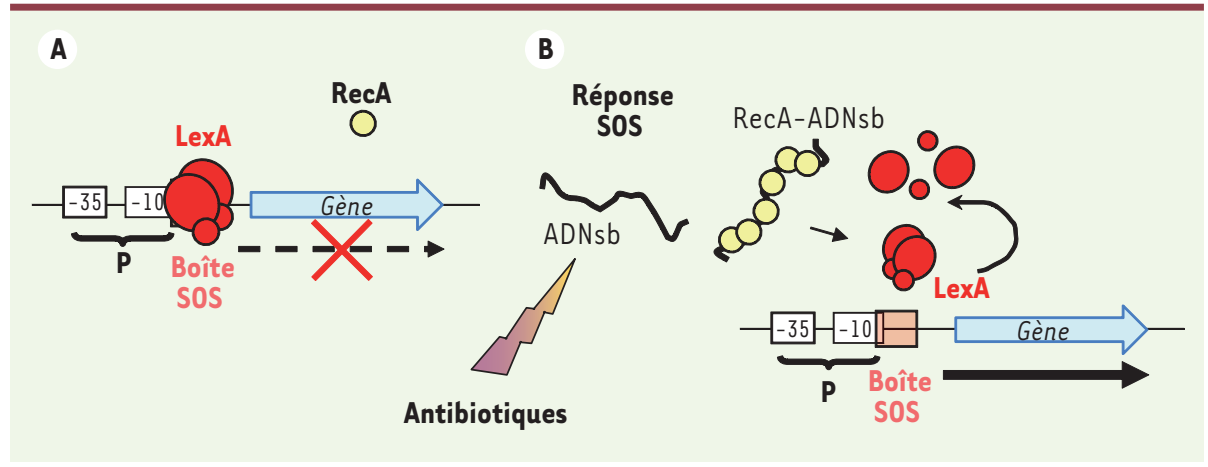

Figure 1. Réponse SOS. A. En l'absence de stress, un dimère de protéines LexA est fixé au niveau de la région promotrice du gène du régulon SOS. Ce gène est totalement inhibé ou exprimé à un niveau basal selon l'affinité de liaison de LexA à son site de fixation (boîte SOS). B. Lors d'un stress provoquant des dommages à I'ADN la protéine RecA interagit avec I'ADN simple brin (ADNsb) et le filament nucléoprotéique formé induit l'autoprotéolyse de LexA libérant le promoteur du gène ainsi exprimé.

deux composants VanS/VanR, qui régule l'expression du cluster de gènes van impliqués dans la résistance des entérocoques à la vancomycine, est activé en réponse à un signal déclenché par la présence de glycopeptides dans le milieu [4]. De même, la tétracycline (TCN) peut induire le transfert de transposons hébergeant des gènes de résistance à cet antibiotique [5].

Depuis une quinzaine d'années, des études ont aussi montré que certains antibiotiques pouvaient favoriser l'acquisition de résistances via l'induction d'un mécanisme impliqué dans l'adaptation des bactéries aux conditions drastiques de l'environnement : la réponse SOS.

\section{La réponse SOS}

La réponse SOS est un mécanisme de régulation qui permet l'adaptation et l'évolution de la bactérie quand les conditions environnementales l'exigent. C'est un système conservé, induit à la suite de stress causant des dommages à l'ADN, et qui est essentiel à la réparation de I'ADN et à la reprise de la réplication. La réponse SOS est régulée par deux acteurs principaux : le répresseur LexA et l'activateur RecA. En période d'homéostasie, LexA est fixé à ses sites de fixation (boîtes SOS) situés dans la région promotrice des gènes du régulon SOS (Tableau I), empêchant ainsi leur expression $[6,7]$. RecA, le senseur du système SOS, se fixe aux fragments d'ADN simple brin (ADNsb) générés quand le génome de la bactérie est endommagé ou que la réplication est interrompue suite à des dommages à l'ADN. La fixation de RecA à l'ADNsb conduit à la formation d'un filament nucléoprotéique, RecA-ADNsb, qui va entraîner le clivage de LexA par autoprotéolyse avec pour conséquence la dérépression du régulon SOS (Figure 1). L'expression des gènes SOS se met en place selon une dynamique précise [8] et dépend aussi du niveau du stress induisant la réponse SOS. Ce système permet de faire agir chaque acteur de la réponse SOS au moment opportun et de manière proportionnelle aux dommages subis.

Le régulon SOS est essentiellement composé de protéines impliquées dans la réparation de l'ADN, comme les excinucléases, hélicases, et recombinases ou ADN polymérases de translésion². Ces dernières ( $A D N$ pol II, IV et $V$ ) n'ont pas d'activité proof-reading et introduisent des erreurs lors des réparations de I'ADN, facilitant ainsi l'émergence de mutations et l'adaptation des bactéries [9].

\section{Antibiotiques induisant la réponse SOS}

De nombreux agents exogènes ou endogènes peuvent induire la réponse SOS, que ce soit de façon directe (via un dommage à l'ADN) ou indirecte [6] (Figure 2). Plusieurs antibiotiques, comme la ciprofloxacine (CIP), l'ampicilline (AMP) ou le triméthoprime (TMP), sont connus pour induire directement, à des concentrations sous-inhibitrices, la réponse SOS chez divers microorganismes [10, 11]. Différentes études montrent que l'induction (ou le niveau d'induction) de la réponse SOS par les antibiotiques semble dépendre de la bactérie et de l'antibiotique. En effet, les aminoglycosides ( $A G$ ), la TCN et le chloramphénicol (CAM) induisent la réponse SOS chez Vibrio cholerae mais pas chez Escherichia coli alors que d'autres molécules (CIP, AMP, TMP) sont inductrices dans les deux espèces [11]. De même, la surexpression de gènes du régulon SOS chez Pasteurella multocida a été observée après traitement par l'enrofloxacine (quinolone) mais pas par l'amoxicilline [12]. Il est donc difficile d'établir des généralités sur les capacités d'induction de la réponse SOS par tel ou tel antibiotique chez telle ou telle bactérie.

Certains antibiotiques (fluoroquinolones [FP], AG, métronidazole, $\beta$-lactamines) peuvent aussi induire indirectement la réponse SOS par production de dérivés réactifs de l'oxygène (ROS) qui induisent chez la bactérie un stress oxydatif [13]. Les ROS, comme le superoxyde ou les radicaux hydroxyles, vont provoquer des dommages

\footnotetext{
${ }^{2}$ « La synthèse de translésion (TLS) est le processus par lequel le réplisome parvient à franchir des lésions au niveau de la fourche de réplication. Le type de lésion franchie peut être une base modifiée, un dimère de thymidines, ou toute autre lésion pas trop encombrante et ne bloquant pas l'hélicase. Ce franchissement évite un arrêt prolongé de la fourche quand la lésion se trouve en face du brin leader, mais au prix de mutations. Cette mutagenèse est due à la faible fidélité des ADN polymérases de translésion (polymérases TLS) qui ont la charge de synthétiser de I'ADN en face des lésions, les polymérases réplicatives en étant incapables. En effet, le site actif des polymérases TLS, plus lâche que celui des ADN polymérases réplicatives, leur permet de prendre en charge des bases modifiées. La contrepartie est la probabilité beaucoup plus importante d'erreurs d'appariement » (source : thèse Samuel Buchsbaum, ENS Lyon, 2006).
} 


\begin{tabular}{|c|c|c|}
\hline Gène & Fonction/produit & Site de fixation de LexA* \\
\hline \multicolumn{3}{|c|}{ Réplication et réparation } \\
\hline polB & ADN polymérase II & GACTGTATAAAACCACAGCC \\
\hline $\operatorname{din} B$ & ADN polymérase IV & CACTGTATACTTTACCAGTG \\
\hline umuDC & ADN polymérase $V$ (UmuC) ; UmuD’ forme un complexe avec UmuC & TACTGTATATAAAAACAGTA \\
\hline$u v r B$ & Réparation de l'ADN par excision ; nucléase d'excision sous-unité B & AACTGTTTTTTTATCCAGTA \\
\hline uvrA & Réparation de l'ADN par excision ; nucléase d'excision sous-unité A & TACTGTATATTCATTCAGGT \\
\hline cho (ydjo) & Réparation de l’ADN par excision ; endonucléase & CACTGGATAGATAACCAGCA \\
\hline $\operatorname{rec} N(1)$ & Réparation de l'ADN par recombinaison & TACTGTATATAAAACCAGTT \\
\hline $\operatorname{recN}(2)$ & Réparation de l'ADN par recombinaison & TACTGTACACAATAACAGTA \\
\hline $\operatorname{recN}(3)$ & Réparation de l'ADN par recombinaison & TAATGGTTTTTCATACAGGA \\
\hline $\operatorname{ruv} A B$ & Réparation de l'ADN par recombinaison; hélicase & CGCTGGATATCTATCCAGCA \\
\hline uvrD & Réplication de l'ADN, réparation de mismatch ; hélicase II & ATCTGTATATATACCCAGCT \\
\hline $\operatorname{din} G$ & Hélicase ATP-dépendante & TATTGGCTGTTTATACAGTA \\
\hline$s s b$ & Réplication de l'ADN, recombinaison & ACCTGAATGAATATACAGTA \\
\hline$s b m C$ & Inhibiteur de l'ADN gyrase & TACTGTATATAAAAACAGTA \\
\hline \multicolumn{3}{|c|}{ Régulateur de la réponse SOS } \\
\hline $\operatorname{lexA} / \operatorname{din} F(1)$ & Répresseur du régulon SOS & TGCTGTATATACTCACAGCA \\
\hline lexA/dinF (2) & Répresseur du régulon SOS & AACTGTATATACACCCAGGG \\
\hline $\operatorname{rec} A$ & Clivage du répresseur, mutagenèse et recombinaison homologue & TACTGTATGCTCATACAGTA \\
\hline \multicolumn{3}{|l|}{ Division cellulaire } \\
\hline sulA & Inhibition de la division cellulaire & TACTGTACATCCATACAGTA \\
\hline ftsk & Protéine essentielle à la division cellulaire & TCCTGTTAATCCATACAGCA \\
\hline \multicolumn{3}{|l|}{ Toxine/antitoxine } \\
\hline tis $A B(y s d A B)$ & TisB : peptide toxique induit par la réponse SOS & TACTGTTTATTTATACAGTA \\
\hline syme (yjiW) & Protéine toxin-like de la réponse SOS & TACTGATGATATATACAGGT \\
\hline hokE & Homologue des gènes hok (host killing) ; interrompu par IS186 & САCTGTATAAATAAACAGCT \\
\hline \multicolumn{3}{|l|}{ Autres fonctions } \\
\hline $\operatorname{dinl}$ & Protéine I, inductible par dommages à l'ADN & ACCTGTATAAATAACCAGTA \\
\hline ydjM & Protéine de membrane interne (prédiction) & TACTGTACGTATCGACAGTT \\
\hline$p c s A(\operatorname{din} D)$ & Protéine inductible par dommages à l'ADN & AACTGTATATAAATACAGTT \\
\hline yebG & Protéine conservée régulée par LexA & TACTGTATAAAATCACAGTT \\
\hline $\operatorname{din} \varrho$ & Protéine inductible par dommages à l'ADN & TACTGTATGATTATCCAGTT \\
\hline $\operatorname{din} S$ & Protéine conservée de l'IS150 & AGCTGTATTTGTCTCCAGTA \\
\hline$y b f \varepsilon$ & Protéine régulée par LexA & AACTGATTAAAAACCCAGCG \\
\hline molR & Régulateur du métabolisme du molybdate & AACTGGATAAAATTACAGGG \\
\hline \multicolumn{2}{|l|}{ Consensus boîte SOS } & TACTGTATATATATACAGTA \\
\hline
\end{tabular}

Tableau I. Gènes chromosomiques régulés par LexA et séquence de leur boîte SOS. * Les parties soulignées dans les séquences LexA de dinG et $\operatorname{recN}(3)$ montrent qu'elles diffèrent du motif consensus « CTG ».

à l'ADN, ce qui pourra alors activer la réponse SOS et ainsi conduire à l'apparition de résistances.

\section{Réponse SOS et acquisition ou expression de la résistance}

Les liens entre réponse SOS et résistances aux antibiotiques peuvent être dus :
- à une augmentation de la fréquence de mutations suite à l'activation de la réponse SOS, pouvant conduire à l'apparition de résistances aux antibiotiques (imperméabilité, modification de cible, etc.) [11, 14] ; - à l'expression de gènes de résistance normalement réprimés par LexA ;

- à des transferts d'éléments génétiques hébergeant des gènes de résistance. 


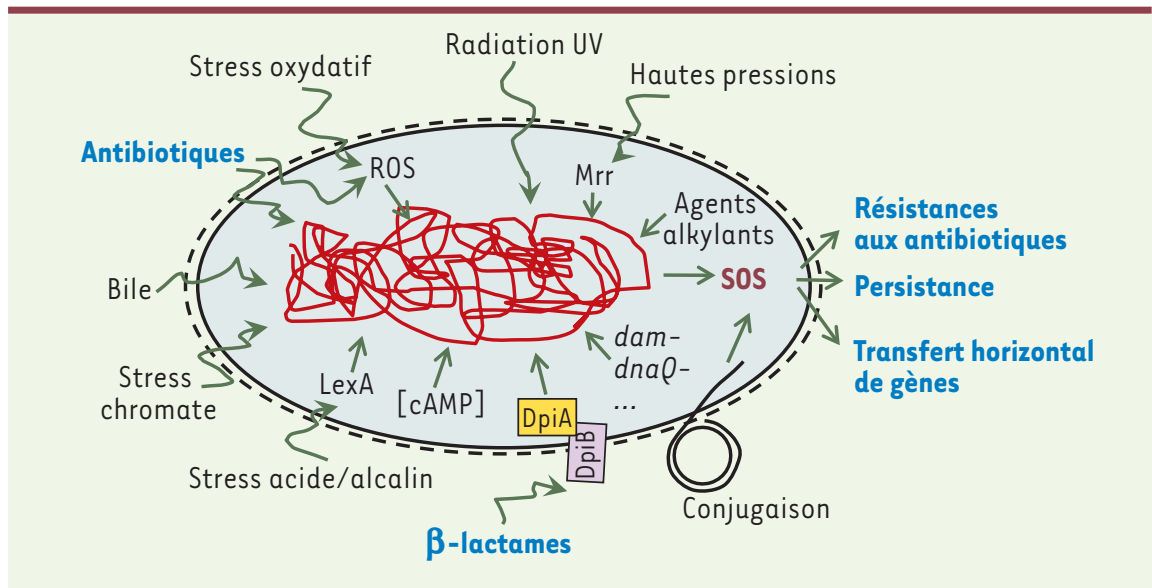

Figure 2. Signaux et mécanismes induisant la réponse SOS. Liste non exhaustive de stress endogènes et exogènes pouvant induire la réponse SOS via des dommages à l'ADN de façon directe ou indirecte (dans ce cas l'intermédiaire lorsqu'il est connu est indiqué). Les réponses impliquant la résistance aux antibiotiques qui sont activées par la réponse SOS sont indiquées. ROS : espèces réactives à l'oxygène (d'après [6]).

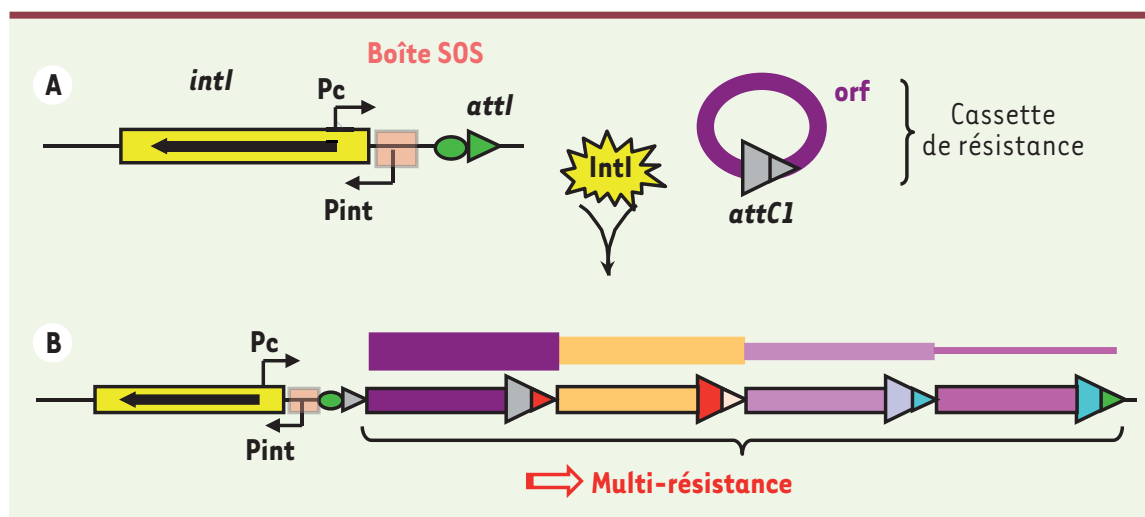

Figure 3. Intégrons de résistance aux antibiotiques. A. Plate-forme structurelle de l'intégron ; intl : gène codant l'intégrase ; Pint : promoteur de l'intégrase ; Pc : promoteur des cassettes de gène ; attl et attC : sites de recombinaison spécifique. $\boldsymbol{B}$. Intégron de multirésistance. Plus une cassette est proche du promoteur Pc, plus elle est exprimée fortement.

Nous illustrons ci-après par des exemples les deux derniers mécanismes.

\section{Induction de l'expression de gènes de résistance :} gènes qnrB et résistance aux quinolones

La résistance aux quinolones, antibiotiques à large spectre, résulte : (1) de mutations de gènes chromosomiques codant pour les topoisomérases, cibles des quinolones, et/ou de gènes régulateurs de protéines de la membrane externe et de pompes d'efflux [15] ; (2) de l'acquisition de gènes plasmidiques tels que les gènes qnr qui protègent la gyrase de l'inhibition par les quinolones, le gène aac6'- $\mathrm{lb}-\mathrm{Cr}$ qui modifie l'agent antimicrobien, ou les gènes qepA ou oqx $A B$ codant pour une pompe d'efflux [16]. Il existe plusieurs familles de gènes qnr, les gènes qnrA, $B$ et $S$ sont très répandus. Nous avons récemment identifié un site de fixation de LexA dans la région promotrice des allèles de la famille qnrB, et avons montré que l'expression des gènes qnr $B$ était régulée directement par la réponse SOS via LexA et RecA et induite par la CIP [17]. Ainsi, la CIP induit directement l'expression d'un gène conférant une résistance vis-à-vis de sa propre action. Aucune régulation par la réponse SOS n'a été observée à ce jour pour les autres déterminants qnr.

\section{Induction de la capture de gènes de résistance par les intégrons}

Les intégrons sont des systèmes génétiques permettant la capture et l'expression de gènes sous forme de cassettes [18]. Les intégrons sont composés d'une plateforme fonctionnelle qui comprend : un gène codant une intégrase spécifique de sites (intl), un site de recombinaison attl et un promoteur Pc permettant l'expression des cassettes (Figure 3). Les intégrons de résistance contiennent des cassettes codant pour des résistances à la plupart des familles d'antibiotiques connus avec plus de 130 cassettes identifiées à ce jour ${ }^{3}$. Ils sont en général portés par des plasmides ou des ICE (integrative conjugative element), ce qui permet leur dissémination efficace. Les cassettes sont constituées d'un cadre ouvert de lecture en général exempt de promoteur et d'un site de recombinaison spécifique attC. Lorsque plusieurs cassettes sont contenues dans le même intégron, elles sont toutes exprimées à partir du promoteur Pc comme dans un opéron. Plus la cassette est proche de Pc plus elle sera exprimée fortement (Figure 3). Les cassettes sont intégrées ou excisées par recombinaison spécifique catalysée par l'intégrase Intl. عn collaboration avec deux autres équipes (D. Mazel, Institut Pasteur, Paris ; J. Barbé, Barcelone, Espagne), nous avons récemment montré in vitro que l'expression de l'intégrase était sous la régulation de la réponse SOS induite par la mitomycine, la CIP et le TMP chez $\varepsilon$. coli et $V$. cholerae, ainsi que par l'AMP chez $V$. cholerae [19]. Ceci implique que l'acquisition de nouvelles cassettes de résistance et/ou le

\footnotetext{
${ }^{3}$ http://integrall.bio.ua.pt/
} 
réarrangement de cassettes existantes dans l'intégron sont induits lors de l'activation de la réponse SOS par les antibiotiques. Une des conséquences est qu'une bactérie peut paraître sensible à un antibiotique alors qu'elle possède la cassette permettant la résistance. En effet, cette cassette peut être dans une position éloignée de Pc et donc peu ou pas exprimée. Sous la pression de sélection exercée par l'antibiotique, l'intégrase sera exprimée via la réponse SOS et pourra réorganiser l'ordre des cassettes permettant ainsi un rapprochement de la cassette du promoteur $\mathrm{Pc}_{\mathrm{c}}$ et donc son expression. Ceci vient d'être observé in vivo dans le cas de la pression de sélection exercée par le métronidazole [20]. En l'absence de stress, le réseau de cassettes est stable et l'intégron n'intégre pas de nouvelles cassettes de résistance.

\section{Induction de la dissémination de gènes de résistance}

Parmi les éléments génétiques mobiles impliqués dans le transfert de gènes de résistance, les plasmides et les ICE ont été très étudiés et sont fortement impliqués dans la dissémination de la résistance. Ce sont des éléments dont le transfert est possible entre bactéries via le processus de conjugaison bactérienne. STX est un ICE trouvé chez $V$. cholerae qui porte des gènes de résistance au CAM, au sulfaméthoxazole, au TMP et à la spectinomycine. II a été montré que l'élément SXT pouvait être transféré à la suite d'un traitement par la CIP de la bactérie hébergeant SXT, selon une voie similaire à la régulation LexA/RecA de la réponse SOS [21]. Dans l'élément SXT, les gènes impliqués dans le transfert sont réprimés par la protéine SetR. Lors de l'induction de la réponse SOS, RecA est activée et entraîne l'autoclivage de SetR, ce qui libère alors les promoteurs des gènes setC et $\operatorname{set} D$, activateurs transcriptionnels des gènes du transfert conjugatif et d'intégration de l'élément SXT. L'élément STX peut ainsi être transféré à un nouvel hôte et ses gènes de résistances peuvent être disséminés.

Par ailleurs, il a été récemment montré que les transferts horizontaux de gènes par conjugaison et transformation induisaient aussi la réponse SOS chez les bactéries à Gram négatif. Ainsi, certains plasmides chez $\varepsilon$. coli et $V$. cholerae, mais aussi l'élément SXT chez $V$. cholerae, sont capables d'induire, via le processus de conjugaison (même s'il est abortif), la réponse SOS dans la bactérie réceptrice ; si cette dernière contient un intégron, l'intégrase est alors exprimée [22]. De même, le processus de transformation induit la réponse SOS et l'expression de l'intégrase d'intégron chez des souches naturellement compétentes de V. cholerae [23]. En effet, lors des processus de conjugaison ou de transformation, le transfert se fait sous forme d'ADNsb permettant l'activation de la réponse SOS.

\section{Induction de la formation de bactéries persistantes}

Les bactéries peuvent résister à un traitement antibiotique sans acquérir de résistance stable (pas de modifications génétiques et effet transitoire) ; dans ce cas on parle de bactéries persistantes [24]. Ces bactéries, dites dormantes, sont caractérisées par un ralentissement de leur métabolisme et sont donc peu sensibles aux actions des antibiotiques actifs sur les bactéries en phase de croissance. Cette propriété résulte de plusieurs mécanismes indépendants et parallèles, dont la réponse SOS [24]. II a été récemment montré que la CIP induisait la formation de bactéries persistantes en réponse à l'induction, sous l'action de la voie SOS, de la toxine TisB chez $\varepsilon$. coli [25]. Tis $B$, toxine du couple toxine/antitoxine tis $A B /$ ist $R$, est un petit peptide hydrophobe qui se lie à la membrane interne et interrompt la force protomotrice, conduisant à une chute des niveaux d'ATP et par conséquent à un état de dormance de la bactérie [25].

Le biofilm est un mode de vie bactérien connu pour sa grande résistance aux traitements antibiotiques. Les bactéries dormantes représenteraient au sein d'un biofilm les individus résistants. Bien que plusieurs antibiotiques (CIP, tobramycine et TCN) augmentent la formation de biofilm par Pseudomonas aeruginosa [26], il n'a toutefois pas été clairement démontré à ce jour que la réponse SOS pouvait jouer un rôle dans l'induction de la formation de biofilm bactérien.

\section{Nouvelles approches de lutte contre la résistance aux antibiotiques}

Pour pallier le manque de nouveaux antibiotiques, de nouvelles approches de lutte antibactérienne sont nécessaires. Plutôt que de cibler des gènes essentiels à la survie des bactéries, il pourrait être intéressant de rechercher des drogues permettant par exemple d'atténuer la virulence ou de potentialiser l'effet d'antibiotiques existants. Ainsi, le concept de «thérapie adjuvante » a été récemment évoqué [27]. La découverte du rôle important de la réponse SOS dans l'expression, l'acquisition et la dissémination de résistances aux antibiotiques chez les bactéries ainsi que dans la formation de bactéries persistantes tolérantes, ouvre de nouvelles de pistes de recherche pour la lutte contre les résistances aux antibiotiques.

L'activation de la réponse SOS se traduit par la levée de l'inhibition par LexA des gènes du régulon SOS sous l'action de RecA. Les protéines LexA et RecA sont des cibles potentielles pour bloquer ce système. Les travaux de Cirz et al [28] ont montré qu'en interférant avec l'autoprotéolyse de LexA, il était possible de rendre une souche d' $\varepsilon$. coli inapte à développer une résistance à la ciprofloxacine in vitro et in vivo. Ceci s'explique par une diminution de la fréquence d'apparition de mutations entraînant la résistance, une conséquence de la noninduction de l'expression des polymérases de translésions du régulon SOS [28]. Plus récemment, le même type d'observations a été fait avec l'utilisation d'un bactériophage non lytique, $M 13 \mathrm{mpl}$, modifié afin d'y induire la surproduction d'un variant non clivable de LexA, $\varphi_{\text {lexA3. }}$. La présence du bactériophage $\varphi_{\text {lexA3 }}$ diminue le développement de résistances après incubation des bactéries avec des concentrations sous-inhibitrices d'ofloxacine (OF) [29]. Plus intéressant encore, les résultats obtenus 
dans cette étude montrent aussi qu'ajouter le bactériophage $\varphi_{\text {lexA3 }}$ au traitement antibiotique (OF, gentamicine ou AMP) augmente de façon significative l'activité bactéricide des $F(, A G$ et $\beta$-lactamines testée sur une souche d' $\varepsilon$. coli in vitro [29]. De même, ce bactériophage accroît in vitro l'effet de la $\mathrm{FP}$ sur une souche d' $\varepsilon$. coli résistante aux quinolones (mutation gyrAl11), et in vivo les chances de survie d'une souris infectée par une souche d'E. coli et traitée par OF (d'un facteur d'environ 4). Ces résultats sont très prometteurs pour le développement d'adjuvants aux antibiotiques qui potentialiseraient leurs effets. Toutefois l'utilisation de bactériophages en clinique pose quelques problèmes en raison de la possibilité de persistance de particules lysogéniques chez le patient. D'autres approches visant à rechercher des petites molécules inhibitrices ou peptides inhibiteurs de RecA sont aussi en développement [30].

\section{Conclusion}

Il semble clair aujourd'hui que l'utilisation des antibiotiques doit être réfléchie. Leur capacité à induire des réponses physiologiques globales face à différents stress et notamment la réponse SOS, en fait des armes à double tranchant. D'une part, ils permettent d'éradiquer les bactéries lors d'une infection, mais en même temps ils favorisent l'apparition, l'acquisition ou la dissémination de résistances, non seulement à leur propre action, mais aussi à celle d'autres antibiotiques, conduisant à la formation de bactéries multirésistantes. Tous les antibiotiques ne semblent pas avoir la même capacité à induire la réponse SOS, la ciprofloxacine étant l'un des plus actifs. Une meilleure connaissance de leurs effets sur les réponses physiologiques bactériennes permettra de mieux cibler leur utilisation et éventuellement d'identifier de nouvelles cibles potentielles pour le développement de nouveaux moyens de lutte contre la résistance aux antibiotiques. $\diamond$

\section{SUMMARY}

Resistance acquisition via the bacterial SOS response :

the inducive role of antibiotics

After the euphoria of the antibiotic discovery and their tremendous action on bacterial infections outcomes, arrives a period of fear with the continuous emergence of bacteria that are resistant to almost all antibiotic treatments. It is becoming essential to better understand antibiotic resistance mechanisms to find new approaches to prevent the worldwide problem of multiresistance. The role of antibiotics on the direct induction of resistance acquisition is known. Recent studies have shown that some antibiotics, by inducing the bacterial SOS response, global repair response after DNA damages, are involved on a broader level in the induction, acquisition and dissemination of resistances in bacteria. We discuss here the role of antibiotics in resistance acquisition via the SOS response through several examples and the interest of identifying the SOS response regulators as the future targets of new families of antimicrobial molecules. $\diamond$

\section{CONFLIT D'INTÉRÊTS}

Les auteurs déclarent n'avoir aucun conflit d'intérêts concernant les données publiées dans cet article.

\section{RÉFÉRENCES}

1. Tremolieres F. Quand le miracle antibiotique vire au cauchemar. Med Sci (Paris) $2010 ; 26: 925-9$.

2. Nordmann P. Résistance aux carbapénèmes chez les bacilles à Gram négatif. Med Sci (Paris) $2010 ; 26: 950-9$.

3. Guillemot D. Antibiotic use in humans and bacterial resistance. Curr Opin Microbiol $1999 ; 2: 494-8$.

4. Depardieu F, Podglajen I, Leclercq R, et al. Modes and modulations of antibiotic resistance gene expression. Clin Microbiol Rev $2007 ; 20$ : 79-114.

5. Courvalin P, Trieu-Cuot P. Minimizing potential resistance: the molecular view. Clin Infect Dis 2001 ; 33 (suppl 3) : S138-46.

6. Erill I, Campoy S, Barbe J. Aeons of distress: an evolutionary perspective on the bacterial SOS response. FEMS Microbiol Rev $2007 ; 31: 637-56$.

7. Kelley WL. Lex marks the spot: the virulent side of SOS and a closer look at the LexA regulon. Mol Microbiol $2006 ; 62: 1228-38$.

8. Friedman N, Vardi S, Ronen M, et al. Precise temporal modulation in the response of the SOS DNA repair network in individual bacteria. PLOS Biol $2005 ; 3:$ e 238 .

9. Friedberg $\varepsilon C$, Walker GC, Siede W, et al. DNA repair and mutagenesis. Washington DC: American society of Microbiology Press, 2006.

10. Shaw KJ, Miller N, Liu X, et al. Comparison of the changes in global gene expression of Escherichia coli induced by four bactericidal agents. J Mol Microbiol Biotechnol $2003 ; 5$ : 105-22.

11. Baharoglu Z, Mazel D. Vibrio cholerae triggers SOS and mutagenesis in response to a wide range of antibiotics, a route towards multi-resistance. Antimicrob Agents Chemother $2011 ; 55$ : 2438-41.

12. Nanduri B, Shack LA, Burgess SC, Lawrence ML. The transcriptional response of Pasteurella multocida to three classes of antibiotics. BMC Genomics $2009 ; 10$ (suppl 2) : S4.

13. Kohanski MA, DePristo MA, Collins JJ. Sublethal antibiotic treatment leads to multidrug resistance via radical-induced mutagenesis. Mol Cell $2010 ; 37$ : 311-20.

14. Gillespie SH, Basu S, Dickens AL, et al. Effect of subinhibitory concentrations of ciprofloxacin on Mycobacterium fortuitum mutation rates. J Antimicrob Chemother $2005 ; 56: 344-8$.

15. Jacoby GA. Mechanisms of resistance to quinolones. Clin Infect Dis $2005 ; 41$ (suppl 2) : S120-6.

16. Rodriguez-Martinez JM, Cano ME, Velasco C, et al. Plasmid-mediated quinolone resistance: an update. J Infect Chemother $2011 ; 17: 149-82$.

17. Da Re S, Garnier F, Guerin $\varepsilon$, et al. The SOS response promotes $q$ nr $B$ quinolone-resistance determinant expression. EMBO Rep 2009; $10: 929-33$

18. Cambray G, Guerout AM, Mazel D. Integrons. Annu Rev Genet 2010 ; 44 : 141-66.

19. Guerin $\varepsilon$, Cambray G, Sanchez-Alberola N, et al. The SOS response controls integron recombination. Science 2009 ; 324 : 1034.

20. Hocquet D, Llanes C, Thouverez M, et al. A Pseudomonas aeruginosa clinical isolate with antibiotic resistance promoted by the SOS response in a patient. ASM $111^{\text {th }}$ general meeting. New Orleans, Louisiana, 2001.

21. Beaber JW, Hochhut B, Waldor MK. SOS response promotes horizontal dissemination of antibiotic resistance genes. Nature $2004 ; 427$ : $72-4$.

22. Baharoglu Z, Bikard D, Mazel D. Conjugative DNA transfer induces the bacterial SOS response and promotes antibiotic resistance development through integron activation. PLoS Genet $2010 ; 6$ : e1001165.

23. Baharoglu Z, Krin $\varepsilon$, Mazel D. Transformation-induced SOS regulation and carbon catabolite control of the V. cholerae integron integrase: connecting environment and genome plasticity. J Bacteriol 2012 (sous presse).

24. Lewis K. Persister cells. Annu Rev Microbiol 2010 ; 64 : 357-72.

25. Dorr T, Vulic M, Lewis K. Ciprofloxacin causes persister formation by inducing the TisB toxin in Escherichia coli. PLOS Biol $2010 ; 8$ : e1000317.

26. Linares JF, Gustafsson I, Baquero F, Martinez JL. Antibiotics as intermicrobial signaling agents instead of weapons. Proc Natl Acad Sci USA 2006; 103 : 19484-9.

27. Kalan L, Wright GD. Antibiotic adjuvants: multicomponent anti-infective strategies. Expert Rev Mol Med 2011 ; 13 : e5.

28. Cirz RT, Chin JK, Andes DR, et al. Inhibition of mutation and combating the evolution of antibiotic resistance. PLoS Biol $2005 ; 3$ : el76.

29. Lu TK, Collins JJ. Engineered bacteriophage targeting gene networks as adjuvants for antibiotic therapy. Proc Natl Acad Sci USA 2009; 106 : 4629-34.

30. Sexton JZ, Wigle TJ, He $Q$, et al. Novel Inhibitors of $\varepsilon$. coli RecA ATPase Activity. Curr Chem Genomics $2010 ; 4: 34-42$.

\section{TIRÉS À PART}

M.C. Ploy 